\title{
Effects of User Correlation on Sample Size Requirements
}

\author{
Sarat C. Dass ${ }^{a}$ and Anil K. Jain ${ }^{b}$ \\ ${ }^{a}$ Department of Statistics \& Probability, Michigan State University, E. Lansing, MI, USA; \\ ${ }^{b}$ Department of Computer Science \& Engineering, Michigan State University, E. Lansing, MI, USA
}

\begin{abstract}
Very little work has been done in determining the number of users needed to establish confidence intervals for an error rate of a biometric authentication system. The independence assumption between multiple acquisitions of an individual is too restrictive and is generally not valid. We relax this assumption and present a semi-parametric approach for estimating the within-user correlation using multivariate Gaussian copula models. We describe how to obtain confidence bands for the ROC and present the minimum requirements on the number of users needed to achieve a desired width for the ROC confidence band. Rules of thumb such as the Rule of 3 and the Rule of 30 grossly underestimate the number of users required. The underestimation becomes more severe when the correlation between any two acquisitions increases.
\end{abstract}

Keywords: Biometric authentication, Error estimation, Gaussian copula models, ROC confidence bands.

\section{INTRODUCTION}

Evaluating the performance of a biometric authentication system involves utilizing multiple individuals (subjects) and, for efficiency, performing multiple verification tests for each individual. It is well known that multiple queries corresponding to each individual exhibit a certain degree of dependence (correlation) (see, for example, ${ }^{1-7}$ ). Earlier efforts to incorporate this correlation include the subset bootstrap approach by Bolle et. al ${ }^{5,8}$ to construct confidence regions for the ROC curve in terms of confidence intervals for the FRR and FAR. However, 90\% confidence intervals for the FARs and FRRs will not automatically guarantee a 90\% confidence region for the entire ROC curve. In fact, one can show that 100(1- $\alpha) \%$ level confidence intervals based on $n$ a-priori selected thresholds will only guarantee a confidence level for the ROC curve of at least $100(1-n \alpha) \%$. Thus, the procedure in ${ }^{5}$ cannot give a specific confidence level for the ROC curve when $n$ becomes large (and this is usually the case since we have to report confidence rectangles at various locations of the ROC curve). Schuckers ${ }^{2}$ introduced the beta-binomial family to model the correlation between multiple queries as well as to account for varying FRR and FAR values for different individuals. He showed that the beta-binomial model gives rise to extra variability in the estimates of FRR and FAR due to the non-zero correlation between multiple queries of a subject. However, a limitation of this approach is that it cannot be used to obtain a confidence band for the ROC curve.

We present a semi-parametric approach for investigating the effects of correlation on the reported authentication performance of biometric systems by first modelling the distribution of the observed genuine and impostor similarity scores. We show how to construct confidence bands for the ROC with a specific confidence level, and are able to demonstrate the effect of varying degrees of correlations on the width of the ROC confidence bands. We also present the minimum number of individuals required to achieve a desired width for the ROC confidence bands. Rules of thumb such as the Rule of $3^{9}$ and the Rule of $30^{10}$ grossly underestimate the number of users required to obtain a specific width for the ROC confidence bands. The underestimation becomes more severe as the correlation between any two acquisitions of a subject increases.

\section{PRELIMINARIES}

Let the number of individuals available for testing a given biometric authentication system be $N$. Each of the $N$ individuals contributes $K$ biometric acquisitions (queries) for testing, resulting in a total of $N K$ acquisitions. In the genuine case when user $i$ uses the system claiming himself/herself to be truly user $i$, the query-template pair is taken from the $K$ acquisitions of user $i, Q_{1}, Q_{2}, \ldots, Q_{K}$, say. Avoiding self-match, each query $Q_{k}$ is matched with the remaining $Q_{h}, h \neq k$ resulting in $K-1$ similarity scores $S\left(Q_{k}, Q_{h}\right), h \neq k$. The average of these similarity scores, $\bar{S}\left(Q_{k}\right)=\frac{1}{K-1} \sum_{h \neq k} S\left(Q_{k}, Q_{h}\right)$

Further author information: (Send correspondence to Sarat C. Dass)

Sarat C. Dass: E-mail: sdass@msu.edu

Anil K. Jain: E-mail: jain@cse.msu.edu 
is taken as the query-specific similarity score for the query $Q_{k}$, and will subsequently be denoted by $S_{k}(i, i)$. In this way, $K$ scores, one per template, are generated for user $i$. When the user $i$ is varied from 1 to $N$, a total of $N$ genuine score vectors, each with $K$ components, are generated. When user $i$ uses the system claiming himself/herself to be user $j, j \neq i$ (this is the impostor case), the query-template pair is taken from the $K$ acquisitions of user $i$ and user $j$, respectively, say, $Q_{1}, Q_{2}, \ldots, Q_{K}$ and $T_{1}, T_{2}, \ldots, T_{K}$. Each query $Q_{k}$ is matched with the templates $T_{h}, h=1,2, \ldots, K$ resulting in $K$ similarity scores $S\left(Q_{k}, T_{h}\right), h=1,2, \ldots, K$. The average of these similarity scores, $\bar{S}\left(Q_{k}\right)=\frac{1}{K} \sum_{h=1}^{K} S\left(Q_{k}, T_{h}\right)$, is taken as the query-specific similarity score for the query $Q_{k}$, and will subsequently be denoted by $S_{k}(i, j)$. In this way, $K$ scores are generated for user $i$. When $i$ and $j$ are varied from 1 to $N$ with the restriction that $i \neq j$, a total of $N(N-1)$ impostor score vectors, each with $K$ components, are generated.

When user $i$ claims the identity of user $j$, the system can either accept the claimed identity as true, or reject the claimed identity as false. Thus, for a fixed threshold $\lambda$, user $i$ is accepted (respectively, rejected) as user $j$ at the $k$-th attempt if $S_{k}(i, j)>(\leq) \lambda$. Defining $\mathcal{S}_{0}=\{(i, j): i=j\}$ and $\mathcal{S}_{1}=\{(i, j): i \neq j\}$, the genuine (respectively, impostor) scores are given by observations $\left\{S_{k}(i, j), k=1,2, \ldots, K\right\}$ with $(i, j)$ in $\mathcal{S}_{0}\left(\mathcal{S}_{1}\right)$. We denote the number of elements in $\mathcal{S}_{0}$ and $\mathcal{S}_{1}$ by $N_{0}=N$ and $N_{1}=N(N-1)$, respectively.

Let $\mathcal{S}(i, j)=\left(S_{1}(i, j), S_{2}(i, j), \ldots, S_{K}(i, j)\right)^{T}$ denote the vector of similarity scores for a combination $(i, j)$. Each vector $\mathcal{S}(i, j)$ is an element of $R^{K}$. We further assume that the vectors $\mathcal{S}(i, j)$ for $(i, j) \in \mathcal{S}_{0}$ (respectively, $\mathcal{S}_{1}$ ) are independent with a common $K$-variate distribution function given by $F_{0}\left(F_{1}\right)$. Note that (i) both $F_{0}$ and $F_{1}$ are multivariate joint distribution functions on $R^{K}$, and (ii) for $h=0,1, F_{h}$ is the common distribution function for every $(i, j) \in \mathcal{S}_{h}$. The $K$ marginal distributions of $F_{0}$ (respectively, $F_{1}$ ) will be denoted by $F_{0, k}\left(F_{1, k}\right)$, for $k=1,2, \ldots, K$. In the next sections, we present the statistical model for $F_{0}$ and its estimation based on the observed genuine scores. The model and estimation procedure for $F_{1}$ based on impostor scores follow in a similar fashion.

\section{COPULA MODELS FOR $F_{0}\left(\right.$ AND $\left.F_{1}\right)$}

Let $H_{1}, H_{2}, \ldots, H_{K}$ be $K$ continuous distribution functions on the real line. Suppose that $H$ is a $K$-dimensional distribution function with the $k$-th marginal given by $H_{k}$ for $k=1,2, \ldots, K$. According to Sklar's Theorem, ${ }^{11}$ there exists a unique function $C\left(u_{1}, u_{2}, \ldots, u_{K}\right)$ from $[0,1]^{K}$ to $[0,1]$ satisfying

$$
H\left(s_{1}, s_{2}, \ldots, s_{K}\right)=C\left(H_{1}\left(s_{1}\right), H_{2}\left(s_{2}\right), \ldots, H_{K}\left(s_{K}\right)\right),
$$

where $s_{1}, s_{2}, \ldots, s_{K}$ are $K$ real numbers. The function $C$ is known as a $K$-copula function that "couples" the onedimensional distributions functions $H_{k}, k=1,2, \ldots, K$ to obtain $H$. Equation (1) can also be used to construct $K$ dimensional distribution functions $H$ whose marginals are the pre-specified distributions $H_{k}, k=1,2, \ldots, K$ : choose a copula function $C$ and define the function $H$ as in (1). It follows that $H$ is a $K$-dimensional distribution function with marginals $H_{k}, k=1,2, \ldots, K$.

The parametric family of copulas we consider in this paper is the $K$-dimensional multivariate Gaussian copulas ${ }^{12}$ given by

$$
C_{R}\left(u_{1}, u_{2}, \ldots, u_{K}\right)=\Phi_{R}^{K}\left(\Phi^{-1}\left(u_{1}\right), \Phi^{-1}\left(u_{2}\right), \ldots, \Phi^{-1}\left(u_{K}\right)\right)
$$

where each $u_{k} \in[0,1]$ for $k=1,2, \ldots, K, \Phi(\cdot)$ is the distribution function of the standard normal, $\Phi^{-1}(\cdot)$ is its inverse, and $\Phi_{R}^{K}$ is the $K$-dimensional distribution function of a random vector $\mathcal{Z}=\left(Z_{1}, Z_{2}, \ldots, Z_{K}\right)^{T}$ with component means and variances given by 0 and 1 , respectively, and with correlation matrix $R$. We define the $(m, n)$-th entry of $R$ as $\rho_{m n}$, where $m, n=1,2, \ldots, K$. Note that $R$ is a positive definite matrix with 1 in the diagonal entries. The distribution function $F_{0}$ will be assumed to be of the form (1) with $H_{k}=F_{0, k}$ for $k=1,2, \ldots, K$, and $C=C_{R_{0}}$, for some correlation matrix $R_{0}$; thus, we have

$$
F_{0}\left(s_{1}, s_{2}, \ldots, s_{K}\right)=C_{R_{0}}\left(F_{0,1}\left(s_{1}\right), F_{0,2}\left(s_{2}\right), \ldots, F_{0, K}\left(s_{K}\right)\right) .
$$

Similarly, the distribution function $F_{1}$ will be assumed to be of the form

$$
F_{1}\left(s_{1}, s_{2}, \ldots, s_{K}\right)=C_{R_{1}}\left(F_{1,1}\left(s_{1}\right), F_{1,2}\left(s_{2}\right), \ldots, F_{1, K}\left(s_{K}\right)\right),
$$

with another correlation matrix $R_{1}$. 


\subsection{Estimation Of $F_{0, k}$ and $R_{0}$}

The marginal distribution functions, $F_{0, k}$, and the correlation matrix $R_{0}$ are unknown and have to be estimated from the observed data of genuine similarity scores, $\left\{\mathcal{S}(i, j):(i, j) \in \mathcal{S}_{0}\right\}$. For $k=1,2, \ldots, K$, the empirical distribution function for the $k$-th marginal given by

$$
E_{0, k}(s)=\frac{1}{N_{0}} \sum_{(i, j) \in \mathcal{S}_{0}} I\left\{S_{k}(i, j) \leq s\right\},
$$

where $I(A)$ is the indicator function of the set $A$. Note that $E_{0, k}(s)=0$ for all $s<s_{\min }$ and $E_{0, k}(s)=1$ for all $s \geq s_{\max }$, where $s_{\min }$ and $s_{\max }$, respectively, are the minimum and maximum of the observations $\left\{S_{k}(i, j):(i, j) \in \mathcal{S}_{0}\right\}$. A partition of $\left[s_{\min }, s_{\max }\right]$ is formed by choosing $L-1$ equidistant points in $\left[s_{\min }, s_{\max }\right]$. For two consecutive partition points defined by $p_{l}=s_{\min }+l \cdot\left(s_{\max }-s_{\min }\right) / L$ and $p_{(l+1)}=s_{\min }+(l+1) \cdot\left(s_{\max }-s_{\min }\right) / L(l$ is an integer with $0 \leq l \leq(L-1))$, the estimate of $F_{0, k}, \hat{F}_{0, k}$ is obtained by interpolation, namely,

$$
\hat{F}_{0, k}(s)=1-\left(1-E_{0, k}\left(p_{l}\right)\right)^{q}\left(1-E_{0, k}\left(p_{l+1}\right)\right)^{1-q}
$$

for $p_{l} \leq s \leq p_{l+1}$ and $q=\left(p_{l+1}-p\right) /\left(p_{l+1}-p_{l}\right)$. To estimate the correlation matrix $R_{0}$, we transform the genuine similarity scores to $\mathcal{Z}(i, j)=\left(Z_{1}(i, j), Z_{2}(i, j), \ldots, Z_{K}(i, j)\right)^{T}$ where

$$
Z_{k}(i, j)=\Phi^{-1}\left(E_{0, k}\left(S_{k}(i, j)\right)\right.
$$

for $k=1,2, \ldots, K$, where $E_{0, k}$ is as given in (5). The mean vector $\overline{\mathcal{Z}}$ is given by $\overline{\mathcal{Z}}=\frac{1}{N_{0}} \sum_{(i, j) \in \mathcal{S}_{0}} \mathcal{Z}(i, j)$ and the covariance matrix is given by $J_{0}=\frac{1}{N_{0}} \sum_{(i, j) \in \mathcal{S}_{0}}(\mathcal{Z}-\overline{\mathcal{Z}}) \cdot(\mathcal{Z}-\overline{\mathcal{Z}})^{T}$. The estimate of the $(m, n)$-th entry of $R_{0}, \hat{\rho}_{0, m n}$, is given by

$$
\hat{\rho}_{0, m n}=\frac{\sigma_{0, m n}}{\sqrt{\sigma_{0, m n} \sigma_{0, m n}}}
$$

where $\sigma_{0, m n}$ is the $(m, n)$-th entry of $J_{0}$.

\section{CONFIDENCE BANDS FOR THE ROC}

Defining $A_{h}(\lambda)=\frac{1}{K} \sum_{k=1}^{K} F_{h, k}(\lambda)$ and $G_{h}(\lambda)=1-A_{h}(\lambda)$ for $h=0,1$, the ROC curve is a plot of $(F A R, G A R) \equiv$ $\left(G_{1}(\lambda), G_{0}(\lambda)\right)$ as $\lambda$ varies. An alternative representation of the ROC curve is $(p, W(p))$, where $W(p)=G_{0}\left(G_{1}^{-1}(p)\right)$. For two fixed numbers $C_{0}$ and $C_{1}$ such that $0 \leq C_{0}<C_{1} \leq 1$, let us consider all $p=F A R$ values that fall in $\left[C_{0}, C_{1}\right]$. A confidence band for the true (claimed) ROC curve of a biometric system at confidence level $100(1-\alpha) \%$ gives a lower bound, $L B(p)$ and an upper bound $U B(p)$ so that for every $p$ in $\left[C_{0}, C_{1}\right]$

$$
L B(p) \leq R O C(p) \leq U B(p)
$$

with probability $100(1-\alpha) \%$. In equation (9), $W(p)$ represents the value of GAR when the FAR is equal to $p$. The numbers $C_{0}$ and $C_{1}$ form the lower and upper bounds of the range of FAR, and will be chosen to cover typical reported values of FAR in biometric applications.

For $h=0,1$, the plug-in estimate of $A_{h}, \hat{A}_{h}$, is given by $\hat{A}_{h}(s)=\frac{1}{K} \sum_{k=1}^{K} \hat{F}_{h, k}(s)$ and $G_{h}$ is estimated by $\hat{G}_{h}(s)=$ $1-\hat{A}_{h}(s)$. This gives an estimate of $W(p), \hat{W}(p)=\hat{G}_{0}\left(\hat{G}_{1}^{-1}(p)\right)$. To construct the $100(1-\alpha) \%$ level confidence interval for the ROC curve in the region of interest $\left[C_{0}, C_{1}\right]$, we form the quantity

$$
z \equiv \max _{C_{0}<p<C_{1}} \sqrt{N_{0}}\left|\sin ^{-1} \sqrt{\hat{W}(p)}-\sin ^{-1} \sqrt{W(p)}\right| .
$$

The function $f(x)=\sin ^{-1} \sqrt{x}$ serves as a variance stabilizing transformation for quantities taking values in $(0,1)$, such as $W(p)$ and $\hat{W}(p) .{ }^{13}$ Assume for the moment that the distribution of $z$ is given. If $z_{1-\alpha}$ denotes the $100(1-\alpha) \%$ percentile of the distribution of $z$, the $100(1-\alpha) \%$ confidence band for the ROC curve is given by

$$
L B(p) \leq W(p) \leq U B(p)
$$


for $C_{0}<p<C_{1}$ with the lower and upper bounds defined as

$$
L B(p)=\left(\sin \left(\sin ^{-1} \sqrt{\hat{W}(p)}-z_{1-\alpha} / \sqrt{N_{0}}\right)\right)^{2}
$$

and

$$
U B(p)=\left(\sin \left(\sin ^{-1} \sqrt{\hat{W}(p)}+z_{1-\alpha} / \sqrt{N_{0}}\right)\right)^{2},
$$

respectively.

Unfortunately, $z_{1-\alpha}$ is difficult to obtain analytically. We find an asymptotic approximation to $z_{1-\alpha}$ for large $N$ based on the multivariate Central Limit Theorem. ${ }^{13}$

\section{EXPERIMENTAL RESULTS}

\subsection{Construction of the ROC}

We used a fingerprint database consisting of fingerprint impressions collected from 50 fingers in our laboratory. ${ }^{14}$ The fingerprint impressions were obtained using an Identix BioTouch USB 200 optical sensor (255x256 images, 380 dpi resolution). 16 impressions of each finger were obtained over 4 days, with 4 impressions taken each day. Thus, we have a total of $N=50$ different fingers with 16 impressions per finger. To avoid the effect of different acquisition days on the correlation, we only considered the first 4 fingerprint impressions taken on the first day. Thus, $K=4$ in our case. A fingerprint similarity score was generated using an asymmetric matcher, described in,${ }^{15}$ based on comparing the minutiae point patterns of a pair of fingerprint impressions. The similarity scores are bounded between 0 and 1000 , and so the order preserving transformation $T\left(S_{k}(i, j)\right)=\log \left\{S_{k}(i, j) /\left(1000-S_{k}(i, j)\right)\right\}$ was used to convert the similarity scores onto the entire real line.

Estimates of the genuine and impostor $4 \times 4$ correlation matrices, $\hat{R}_{0}$ and $\hat{R}_{1}$, are given by

$$
\left(\begin{array}{llll}
1.0 & 0.5 & 0.5 & 0.4 \\
0.5 & 1.0 & 0.7 & 0.6 \\
0.5 & 0.7 & 1.0 & 0.8 \\
0.4 & 0.6 & 0.8 & 1.0
\end{array}\right) \text { and }\left(\begin{array}{cccc}
1.0 & 0.2 & 0.2 & 0.3 \\
0.2 & 1.0 & 0.2 & 0.2 \\
0.2 & 0.2 & 1.0 & 0.3 \\
0.3 & 0.2 & 0.3 & 1.0
\end{array}\right)
$$

respectively. The off-diagonal entries of $\hat{R}_{0}$ and $\hat{R}_{1}$ indicate the degree of correlation between the corresponding row and column dimensions. Comparing the off-diagonal entries of $\hat{R}_{0}$ and $\hat{R}_{1}$, we see that the genuine correlations are much larger than the impostor correlations. One reason for this is that the genuine correlations arise from the same user using the system a multiple number of times. Thus, typical user characteristics (for example, how the finger is placed on the sensor, amount of pressure exerted on the finger, the sitting position, etc.) are reflected in the similarity scores. In the case of impostor scores, a variety of users smooths out this typicality resulting in smaller correlations.

Figure 1 shows the ROC confidence bands based on the (i) non-parametric bootstrap, and (ii) the asymptotic approaches. The resulting upper and lower bounds of the two approaches closely match with each other indicating a good agreement of the semi-parametric model to the true distribution of genuine and impostor similarity scores.

Next, we study the effect of correlation among the multiple impressions of a user on the width of the ROC confidence band. As we require to vary the correlation, this experiment is not possible using real data. Our experiment was based on simulated data from the multivariate Gaussian $K$-copula models with genuine and impostor correlation matrices that have a common correlation parameter for the off-diagonal entries, say $r$. For the genuine case, we selected two values of $r$ : $r_{G}=0$ (independence) and $r_{G}=0.575$ (this corresponds to the average of the 12 off-diagonal elements of the $\hat{R}_{0}$ matrix in (14)). For the impostor case, we selected two values of $r: r_{I}=0$ (independence) and $r_{I}=0.255$ (the average of the 12 off-diagonal elements of the $\hat{R}_{1}$ matrix in (14)). Four pairs for the genuine-impostor correlations, $\left(r_{G}, r_{I}\right)$, were thus obtained: $(0,0),(0,0.255),(0.575,0)$ and $(0.575,0.255)$. The $95 \%$ ROC confidence bands for the four pairs had median widths of $8.70,8.71,10.82$ and 10.97, respectively. Thus, higher correlations between multiple biometric observations result in a wider confidence band for the ROC curve.

Next, we determine the number of users, $N$, required by a system to report a $100(1-\alpha) \%$ ROC confidence band with a width of at most $w$, where $w$ is pre-specified. The values of correlations selected in the simulation study reflect zero 


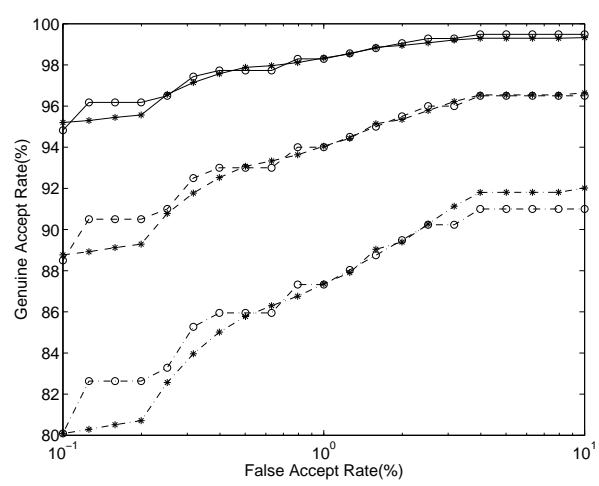

Figure 1. 95\% ROC confidence bands based on (a) the non-parametric ROC band (lines with ' $\circ$ '), and (b) the asymptotic method (lines with '*'). The dashed, solid and dot-dashed lines represent the estimated ROC curves, upper and lower confidence bounds, respectively.

Table 1. Values of $N_{\min }$ for achieving a width of $1 \%$ for the $95 \%$ confidence band. The total number of observations, $N_{\min } K$, is given in parenthesis.

\begin{tabular}{c|cccc} 
Correlation & \multicolumn{4}{|c}{ Number of acquisitions per user, $K$} \\
\cline { 2 - 5 }$r$ & 1 & 2 & 4 & 8 \\
\hline 0.0 & 65,962 & 32,582 & 16,475 & 8,234 \\
& $(65,962)$ & $(65,164)$ & $(65,900)$ & $(65,882)$ \\
0.255 & 65,858 & 36,238 & 20,645 & 13,409 \\
& $(65,858)$ & $(72,476)$ & $(82,580)$ & $(107,272)$ \\
0.575 & 65,399 & 42,036 & 29,418 & 22,498 \\
& $(65,399)$ & $(84,072)$ & $(117,672)$ & $(179,984)$
\end{tabular}

(total independence), moderate and high degrees of correlation between multiple acquisitions per user. Thus, the results of the simulation study can be generalized to real data which exhibit different degrees of correlation. Three choices of the genuine-impostor correlation pairs were considered: $\left(r_{G}, r_{I}\right)=(0,0),(0.255,0.255)$ and $(0.575,0.575)$. The minimum number of users required is given by the formula $N_{\min }=\left[\left(\frac{2 z_{1-\alpha}}{w}\right)^{2}\right]+1$.

Table 5.1 reports $N_{\min }$ for obtaining a $95 \%$ confidence band with $w=1 \%$. We took $C_{0}=0.1 \%$ and $C_{1}=10 \%$. The numbers in parenthesis in Table 5.1 give the total number of observations $N_{\min } K$. In other words, if a biometric authentication system was tested based on $N$ users, where $N$ is chosen according to the entries in Table 5.1, we will be $95 \%$ certain that the true ROC curve will lie in the interval $[\hat{W}-0.5, \hat{W}+0.5]$ for all $0.1 \% \leq F A R \leq 10 \%$. Table 5.1 indicates that as correlation among the multiple impressions of a finger increases for each fixed $K$, the total number of observations needed to achieve the width $w$ for the confidence band increases. The same holds true when $K$ is increased for each fixed non-zero correlation. Thus, in the presence of non-zero correlation, we are better off selecting a larger number of users rather than increasing the number of multiple acquisitions per user. We also obtained the minimum sample sizes determined by the Rule of $3, N_{3},\left({ }^{9}\right)$ and the Rule of $30, N_{30}\left({ }^{10}\right)$. Since both rules were derived for setting up confidence intervals for specific values FAR and GAR (and not confidence bands for a range of FAR and GAR values), we were required to modify them slightly to suit the present case. For the Rule of 3 , we computed the quantity $1-L B\left(p_{l}\right), l=1,2, \ldots, L$ and derived the minimum sample size as $N_{3}=\max _{1 \leq l \leq L} \frac{3}{1-L B\left(p_{l}\right)}$ The smallest sample size based on the Rule of 30 was obtained using the formulas $F R R_{l}=1-W\left(p_{l}\right), e_{l}=\left(\frac{2(1.96) F R R_{l}}{W\left(p_{l}\right)}\right)^{2}$ and, $N_{30}=\max _{1 \leq l \leq L} \frac{e_{l}}{F R R_{l}}$. We found $N_{30}$ ranged between 17,300-17,600 whereas $N_{3}$ was approximately 105 for all values of the correlation $r$ and $K$. Thus, $N_{3}$ and $N_{30}$ grossly underestimate the total number of biometric acquisitions required to achieve a desired width. The underestimation becomes more prominent when significant correlation is present between multiple acquisitions of the biometric templates from a subject. 


\section{SUMMARY \& CONCLUSIONS}

We present a semi-parametric approach for constructing ROC confidence bands and determining the minimum number of users required to achieve a desired width for the ROC confidence band based on multivariate Gaussian copula models. A loss of efficiency is observed (in terms of the total number of acquisitions required) when there is considerable correlation present between multiple acquisitions per user. In this case, we are better off selecting a larger number of users rather than increasing the number of acquisitions per user. Our future work will be to investigate the effects of user correlation on sample size requirements for a larger fingerprint database.

\section{ACKNOWLEDGMENTS}

The authors wish to thank Karthik Nandakumar, Anoop Namboodiri, Arun Ross, Umut Uludag and Yi Chen for their help in conducting experiments. This research is partially supported by the NSF ITR grant 0312646.

\section{REFERENCES}

1. "U. K. Biometrics Working Group. Best practices in testing and reporting performance of biometric devices," 2000. Online: www.cesg.gov.uk/technology/biometrics.

2. M. E. Schuckers, "Using the beta-binomial distribution to assess performance of a biometric identification device," International Journal of Image and Graphics (Special Issue on Biometrics) 3, pp. 523-529, July 2003.

3. J. Wayman, "Technical testing and evaluation of biometric identification devices," in Biometrics: Personal Identification in Networked Society, A. K. Jain, R. Bolle, and S. Pankanti, eds., Kluwer Academic Publishers, 1999.

4. J. Wayman, "Confidence interval and test size estimation for biometric data," in National Biometric Center Collected Works, J. Wayman, ed., pp. 91-95, 2000.

5. R. Bolle, N. Ratha, and S. Pankanti, "Error analysis of pattern recognition systems: The subsets bootstrap," Computer Vision and Image Understanding 93, pp. 1-33, January 2004.

6. J. R. Beveridge, K. She, and B. A. Draper, "A nonparametric statistical comparison of principal component and linear discriminant subspaces for face recognition," in Proc. of the IEEE Conf. on Computer Vision and Pattern Recognition (CVPR 2001), Hawaii, December 2001.

7. R. J. Micheals and T. E. Boult, "Efficient evaluation of classification and recognition systems," in Proc. of the IEEE Conf. on Computer Vision and Pattern Recognition (CVPR 2001), Hawaii, December 2001.

8. R. Bolle, J. Connell, S. Pankanti, N. Ratha, and A. Senior, Guide to Biometrics, Springer, 2004.

9. J. Wayman, "Technical testing and evaluation of biometric identification devices," in National Biometric Center Collected Works, J. Wayman, ed., pp. 67-89, 2000.

10. J. Porter, "On the "30 error criterion"," in National Biometric Center Collected Works, J. Wayman, ed., pp. 51-56, 2000.

11. R. B. Nelsen, An Introduction to Copulas, Springer, 1999.

12. U. Cherubini, E. Luciano, and W. Vecchiato, Copula Methods in Finance, Wiley, 2004.

13. C. R. Rao, Linear Statistical Inference And Its Applications, Wiley, 1991.

14. A. K. Jain, U. Uludag, and A. Ross, "Biometric template selection: a case study in fingerprints," in Proc. AVBPA 2003, Fourth International Conference on Audio- and Video-Based Biometric Person Authentication, Guildford, UK, pp. 335-342, June 2003.

15. A. K. Jain, L. Hong, and R. Bolle, "On-line fingerprint verification," IEEE Transactions on Pattern Recognition and Machine Intelligence 19(4), pp. 302-314, 1997. 